\title{
PERFECTO SIMPLE Y PERFECTO COMPUESTO: DE LA GRAMÁTICA AL DISCURSO*
}

\author{
IGNACIO MORENO-TORRES SÁNCHEZ \\ (Universidad de Málaga)
}

\begin{abstract}
This article is part of a larger project which aims at describing - from a formal semantics perspective- the temporal structure of discourse in Spanish. In this paper we analyse the role that two Spanish tenses - perfecto simple and perfecto compuesto- play in discourse. Using the framework provided by Discourse Representation Theory, we describe the semantics of these two tenses and we propose a representation for both of them. The representation proposed is based on Dowty's extended now theory of the present perfect (English equivalent for Spanish perfecto compuesto). We show that the extended now theory provides a very intuitive way of representing not only perfecto compuesto, but also the perfecto simple and the effect of several adverbials. Finally, we will examine the discourse relations of sentences in which perfecto simple and perfecto compuesto appear.
\end{abstract}

\section{Preliminares}

El presente trabajo se enmarca en un proyecto cuya finalidad es estudiar las relaciones temporales entre enunciados del español así como la función que tienen en el discurso. Para ello partimos del supuesto de que diversos elementos gramaticales - y no solo la información extralingüística — restringen y determinan las posibles relaciones temporales en el discurso.

Aquí nos centramos en dos tiempos verbales, el perfecto simple (PS) y el perfecto compuesto $(\mathrm{PC})$. Estudiaremos en primer lugar sus propiedades semánticas en el nivel oracional y propondremos una representación semántica para las

\footnotetext{
*Agradezco a Joan Busquets y Juan Carlos Moreno Cabrera los comentarios sobre una versión anterior de este trabajo. Como es natural, los errores pendientes son responsabilidad exclusiva del autor.
} 
oraciones con PC y otra para las oraciones con PS; las representaciones obtenidas reflejarán la proximidad de ambos tiempos verbales así como las diferencias entre ambos. A continuación analizamos el comportamiento de ambos tiempos verbales cuando aparecen en secuencias de oraciones (nivel discursivo).

Como marco teórico adoptamos la Teoría de Representación del Discurso (TRD) tal y como se describe en el manual de Kamp y Reyle (1993). La TRD puede considerarse la continuación natural de la semántica de Montague (1974) ya que, conservando los principios de la semántica veritativa, da el salto de la semántica de la oración a la semántica del discurso.

El resto del artículo se estructura como sigue. En primer lugar expondremos brevemente la concepción semántica que aquí seguimos y mostraremos algunos rudimentos de la metodología de trabajo. A continuación repasamos algunas propuestas sobre las relaciones entre PC y PS y formularemos nuestra propuesta de representación para PC y PS; además mostraremos como la representación propuesta sigue siendo válida en presencia de la negación y determinadas expresiones temporales. Por último examinaremos las relaciones temporales desde una perspectiva discursiva.

\section{Principios teóricos}

La TRD asume como concepción semántica la denominada semántica veritativa. La semántica veritativa surge dentro de la filosofía del lenguaje (Tarski, Davidson, etc.). Sus objetivos y su concepción del significado se oponen a la más intuitiva semántica ideacionista. Según la semántica ideacionista «el significado de los elementos léxicos y de las oraciones son ideas localizadas en las mentes de los hablantes» (Moreno Cabrera, 1994, T. II, pág. 311). El objetivo que se plantea la semántica ideacionista es reconstruir tales objetos mentales. Así, para la siguiente oración:

\section{(1) La nieve es blanca}

el semántico ideacionista estudiaría de qué forma podemos construir una representación mental de esta oración a partir de los elementos básicos que la componen. Por el contrario, la semántica veritativa considera prioritario conocer la relación entre lo lingüístico y lo extralingüístico. O sea, considera que lo más importante no es ya conocer la representación de (1) sino saber la verdad o falsedad de tal oración. Y la verdad o falsedad de una expresión linguística sólo la conoceremos si la comparamos con aquello acerca de lo que nos informa. 


\subsection{Metodologia de la semántica veritativa}

Para establecer las condiciones de verdad de una oración debemos:

a) Establecer la aportación de cada uno de los elementos de la oración.

b) Combinar las diferentes aportaciones; para ello obtendremos, como paso intermedio, un árbol sintáctico; el resultado de la combinación será un conjunto de condiciones de verdad.

c) Por último, para comprobar si las condiciones anteriores se cumplen, debemos disponer de un modelo del mundo (una representación del mundo extralingüístico). En un modelo básico encontraremos tan solo una serie de individuos así como relaciones o propiedades de los individuos. Para representar información temporal debemos incluir, además de individuos, instantes y eventualidades.

\section{2. Construcción de representaciones}

En nuestras representaciones y análisis seguiremos la notación y los principios de la Teoría de Representación del Discurso (Kamp y Reyle, 1993). La TRD nos ofrece un algoritmo sistemático para convertir un árbol sintáctico en una serie de condiciones de verdad. El algoritmo se describe con toda claridad en el manual de Kamp y Reyle. Nos limitamos ahora a mostrar un ejemplo. Veamos el árbol sintáctico y la representación obtenida para la siguiente oración.

(2) Luis llegó a las 10
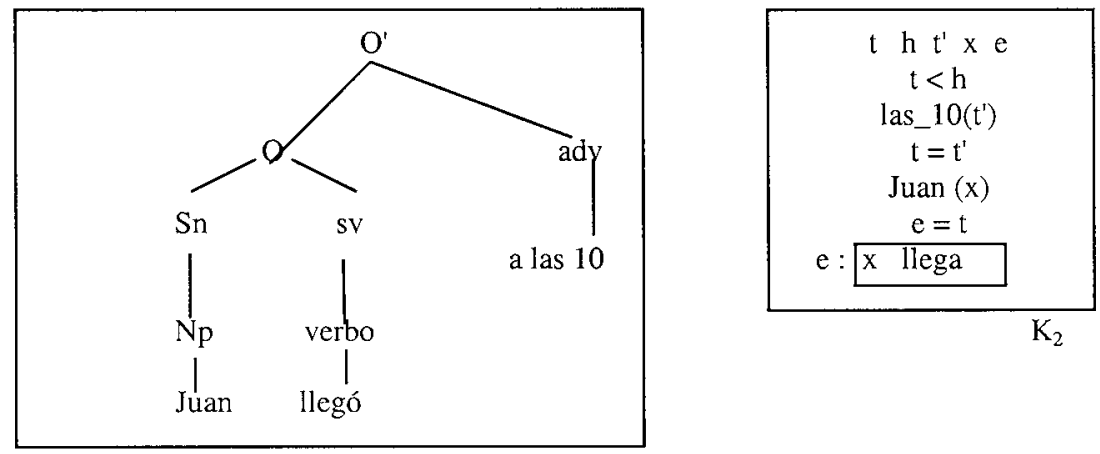

$\mathrm{K}_{2}$ es la representación semántica de (2). Puede parafrasearse de la siguiente forma:

(2) es verdadero si:

a) En un instante $t$ anterior al momento del habla $(h)$

b) que coincide con otro instante $t^{\prime}$ (las 10)

c) ocurre un suceso $e$ (Juan habla) 
O dicho de otra forma, si el la extensión de «llega» en el modelo incluye una terna: (Juan, e, las 10).

\subsection{Tiempo, modo de acción y aspecto ${ }^{I}$}

\section{Tiempo}

Los tiempos gramaticales son un mecanismo mediante el cual los hablantes indican a sus oyentes la posición en el tiempo de los sucesos o hechos de los que hablan. La forma más simple de situar un suceso en el tiempo consiste en indicar si es anterior, posterior o coincidente con el momento del habla. Pero las lenguas nos ofrecen mecanismos más sofisticados. Reichenbach (1947) propuso que los sucesos pueden situarse con respecto a dos momentos: el momento del habla $(h)$ y un instante de referencia $(R)$. El siguiente ejemplo permite ilustrar la propuesta de Reichenbach:

\section{(3) María llegó a las 10. Paco había llegado mucho antes.}

En la segunda oración, el pluscuamperfecto permite expresar relaciones entre:

a) El momento en el que se pronuncia la oración (ahora)

b) Un punto de referencia (las 10, justo cuando María llegó).

c) El suceso de la Ilegada de Paco

Reichenbach propuso que a partir de las relaciones entre momento del habla, el suceso y el instante de referencia se podían describir todas las posibilidades que ofrece la lengua. Sin embargo esto no es así. Por ejemplo, si traducimos el análisis de los tiempos de Rojo $(1974,1990)$ al modelo de Reichenbach (1947) obtenemos dos puntos de referencia; y Kamp y Reyle (1993) no emplean puntos de referencia con algunos tiempos.

Aunque la propuesta de Reichenbach presenta algunas limitaciones, tomamos de este autor la idea básica de que es preciso (y posible) dar forma matemática a las relaciones temporales y describirlas a partir de un conjunto pequeño de elementos; consideramos como objetivo básico identificar cuáles son estas relaciones para el español.

Modo de acción y aspecto

Como apuntábamos antes, mediante cualquier oración podemos describir sucesos. En este trabajo supondremos que los sucesos son de uno de estos tipos:

\footnotetext{
${ }^{1}$ La bibliografía sobre los conceptos que introducimos aquí es abundantísima. Aquí nos limitamos a introducir algunos conceptos que son necesarios para seguir el resto del artículo. Especialmente nos interesa llamar la atención al lector sobre la diferencia entre la interpretación obtenida para una oración y los elementos gramaticales que nos llevan a ella. Esto es, no debe confundirse la naturaleza estativa o eventiva de un verbo con el hecho de que la interpretación de una oración construida con ese verbo sea eventivo o estativo.
} 
estados y eventos ${ }^{2}$. Para saber qué tipo de suceso describe una oración es preciso combinar dos tipos de información: el modo de acción y el aspecto.

El modo de acción es una propiedad del conjunto formado por el verbo y todos sus complementos pero sin determinados morfemas o modificadores aspectuales (como los morfemas verbales o verbos como quedar, acabar, empezar...) y cuantificadores (como el determinante de los SSNN). Las siguientes oraciones comparten un mismo modo de acción:

Juan construyó una casa

Juan está construyendo una casa

Juan empezó a construir una casa

Juan construyó casas

Por su modo de acción podemos clasificar las oraciones como estativas o eventivas. Son estativas las que sirven para describir situaciones que no tienen un final intrínseco (como ser simpático) y eventivas las que sí lo tienen (construir).

La noción de aspecto hace referencia a las diferentes formas en las que se puede presentar un mismo suceso. Por ejemplo se puede presentar desde fuera para verlo como acabado o para llamar la atención sobre sus resultados -aspecto perfectivo, o bien se puede presentar desde dentro como no acabado -aspecto imperfectivo.

Aquel día, Juan corrió los 1000 metros (aspecto perfectivo)

Juan corría a menudo los 1000 metros (aspecto imperfectivo)

Se ha notado ${ }^{3}$ que el aspecto perfectivo tiene dos variantes muy próximas, que llamaremos resultativa y perfecta.

a) Resultativa: se enfoca el estado resultante de una acción (que queda presupuesta). El estado resultante puede deducirse directamente de la acción descrita por el verbo, por lo que hablaremos de resultativos léxicos. En español el aspecto resultativo aparece en perífrasis como:

$$
\begin{aligned}
& \text { quedar }+ \text { participio } \rightarrow \text { quedó agotado } \\
& \text { estar }+ \text { participio } \rightarrow \text { estar roto }
\end{aligned}
$$

b) Perfecta: se presenta un suceso como acabado. En español este aspecto perfecto es el que normalmente asociamos al PC y al PS:

Juan ha roto con María

Juan rompió con María

\footnotetext{
${ }^{2}$ La clasificación de los sucesos o situaciones más conocida es la propuesta por Vendler (1967), quien distingue entre logros, realizaciones, actividades y estados.

${ }^{3}$ Por ejemplo, en relación a los tiempos que aquí tratamos lo mencionan Alarcos (1970) y Carcy (1996).
} 


\section{Interpretación}

Para conocer la interpretación (como estado o evento) de un enunciado debemos calcular el efecto de los operadores aspectuales y cuantificacionales sobre el modo de acción. En nuestro caso las posibilidades serán dos:

a) Si el aspecto es perfecto, la interpretación dependerá sólo del modo de acción:

Juan ha estado en Madrid $\quad \rightarrow$ se describe un estado

Juan ha roto la silla $\quad \rightarrow$ se describe evento

b) Si el aspecto es resultativo, se describe un estado y un suceso previo (del tipo asociado al modo de acción):

Juan ya ha estado en Madrid $\rightarrow$ se describe un estado que da lugar a otro estado

Juan ya ha roto la silla $\longrightarrow$ se describe un evento que da lugar a un estado

\section{Análisis del PC y PS}

El PS es aceptado generalmente como tiempo perfecto y pasado (sirve para describir sucesos como acabados y pasados). Sin embargo, la naturaleza del PC se ha explicado de diversas formas. Examinamos en primer lugar las posibles lecturas de las oraciones construidas con PC. A continuación estudiamos las diferencias entre PC y PS.

\subsection{Lecturas del PC}

Para el PC se ha defendido la existencia de dos lecturas, si bien sólo la perfecta es generalmente aceptada.

-Lectura resultativa del PC

Recientemente García Fernández (1995) ha defendido la existencia de un PC resultativo, que se caracterizaría por lo siguiente:

a) No podemos localizar el evento mediante un temporal, pero sí el estado resultante. De ahí que en:

A las 3 ya ha salido

a las 3 no se refiera al evento sino al resultado.

b) No permiten la interrogación temporal con cuando

* ¿Cuándo ya has llegado?

c) Se combinan difícilmente con verbos estativos, ya que no presuponen un

final o transición: 
* Al ver la fotografía de Juan, María pensó que ya había sido un hijo excelente

d) El sujeto nunca es un agente. De ahí que no acepten tiempos adverbios como «deliberadamente».

No estamos de acuerdo con que sea preciso postular una lectura resultativa para el PC español. Básicamente, en todos los ejemplos que aporta este autor de lectura resultativa aparece el adverbio ya. Ello nos lleva a pensar que la lectura resultativa tal vez sea una propiedad de este adverbio y no del PC. Además, este adverbio también puede aparecer con el perfecto simple en: yo ya estuve allí antes, por lo que no tendría que considerarse una propiedad específica de los tiempos compuestos.

\section{-Lectura perfecta}

La lectura perfecta es la que obtenemos si entendemos que el PC sirve para describir sucesos acabados pero de alguna manera presentes. La encontramos en ejemplos como los siguientes:

Juan te ha llamado por teléfono

Juan ha estado enfermo esta semana

Como veremos más adelante, aún aceptando esta propuesta podemos llegar a dos representaciones diferentes del $\mathrm{PC}^{4}$, la veremos más adelante.

-Otras lecturas

Junto a estas lecturas aspectualmente diferenciadas, se han distinguido diferentes matices asociados típicamente al PC:

a) Experiencial:

Yo he conocido al Presidente de la República

b) Noticias recientes:

\section{Malcom X ha sido asesinado}

Creemos que estos matices se deben a otros factores tales como el estilo o el contexto. Por ejemplo, dado que lo específico del PC es la «relevancia actual», es natural escogerlo para dar noticias («Malcom X ha sido asesinado») o para destacar un hecho personal como relevante; no por ello debemos considerar que se trate de lecturas aspectualmente diferenciadas.

\section{- Conclusión}

Partimos por tanto del supuesto de que sólo hay una lectura del PC: la lectura perfecta.

\footnotetext{
${ }^{4}$ Según sigamos las propuestas de Rojo (1974) o Alarcos (1970).
} 


\subsection{Diferencias entre las lecturas perfectas del PS y PC}

Aunque el PC y el PS sean tiempos perfectos, se diferencian porque el PC se emplea cuando un suceso o algunos de sus efectos siguen siendo relevantes. Sin embargo, no hay acuerdo sobre la forma en que esa relevancia debe formalizarse:

1) Algunos autores (Kamp y Reyle, 1993; Asher y Lascarides, 1993) proponen vincular la relevancia a un estado resultante-pragmático ${ }^{5}$.

2) Otros (como Rojo, 1974, 1990; o Reichenbach, 1947) explican las diferencia entre PC y PS apoyándose en los puntos de referencia específicos de cada tiempo.

Por último, otros autores (Alarcos, 1970; Dowty, 1979) se basan en la noción de presente ampliado.

\subsection{Representación del PC y PS}

\subsubsection{Vigencia del «estado resultante»}

Kamp y Reyle (1993) defienden una interpretación resultativo-pragmática para el PC. Proponen la siguiente representación:

(4) Juan ha llegado

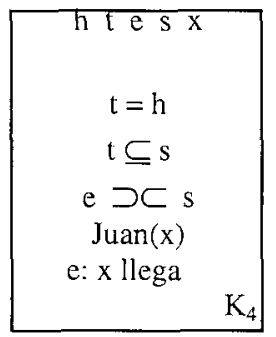

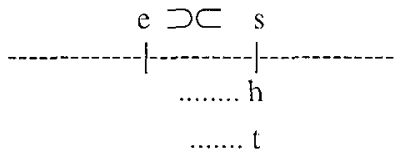

La representación de la izquierda $\left(\mathrm{K}_{4}\right)$ puede leerse:

- Hay un evento $e$ (Juan llega),

- seguido inmediatamente (つС) por un estado $s, \mathrm{y}$

- $s$ se mantiene en $h$ (el momento del enunciado).

El elemento clave de esta propuesta es el estado $s$. Kamp y Reyle (1993) asumen que ese estado no se infiere sólo a partir de información léxica, sino a partir

\footnotetext{
${ }^{5}$ Aunque estos autores aplican sus análisis al francés y al inglés y el PC de estas lenguas - especialmente el del francés- no coincide con el español, nos parece interesante comprobar las posibilidades de esta alternativa. Además, esta forma de diferenciarlos también aparece en nuestra tradición, en particular en Bello (1978).
} 
de una combinación de información léxica y contextual ${ }^{6}$. Así, tales estados no sólo aparecen con realizaciones y logros vendlerianos sino también con estados como Juan ha estado en Madrid. Pero hay diversos argumentos para no emplear esta representación para el español.

a) Lo característico del PC español es que sitúa los eventos en el presente. Sin embargo, lo que Kamp y Reyle sitúan en el presente es el resultado $s$.

b) Esta propuesta resulta problemática al representar negaciones de PC. Veamos un ejemplo:

(5a) Juan se ha gastado todo el dinero

(5b) Juan no se ha gastado todo el dinero

(5b) podría parafrasearse:

1. El evento «gastar todo el dinero» no ha ocurrido y, por tanto, tampoco el estado resultante de tal evento.

2. Se da un estado de cosas como consecuencia de no haber ocurrido el evento «gastar todo el dinero».

Dado que el estado resultante de la afirmativa no es un estado léxico sino pragmático, parece que en la negación deberíamos mantener un estado (o sea, que la paráfrasis correcta sería la segunda). Sin embargo, no queda claro cómo se inicia ese estado debido a que no hay un evento que lo produzca. Esto es, en la oración afirmativa el estado se inicia cuando termina el evento; pero ¿y en la negativa?; dado que no hay evento, ¿cuándo se inicia el estado?

Más adelante veremos cómo podemos obtener representaciones adecuadas para oraciones como (5a) y (5b).

\subsubsection{Punto de referencia actual}

Una segunda aproximación a las diferencias entre PC y PS considera que el hablante describe las eventualidades usando unos puntos de referencia. En concreto Rojo (1974, 1990) y Reichenbach (1947) proponen lo siguiente: 1) la representación del PC incluyen un punto de referencia coincidente con el momento del habla; y 2) la representación del PS o bien no tiene un punto de referencia adicional (Rojo) o bien lo tiene pero coincide con el propio evento (Reichenbach). Desde el punto de vista de una semántica veritativa, estas propuestas resultan problemáticas porque las condiciones de verdad para estas oraciones serían

\footnotetext{
${ }^{6}$ Otros autores, como Vet (1980) hablan de un estado resultante meramente léxico. Esto es, según Vet habría un estado resultante en la representación semántica de Juan ha roto un vaso, pero no en la de $J$ uan ha estudiado matemáticas, debido a que el primer verbo describe un cambio de estado (es un verbo transicional en la terminología de Vet) pero el segundo no.
} 
idénticas; en tal caso para diferenciar oraciones como (5a) y (5b) tendríamos que recurrir a información no semántica ${ }^{7}$ :

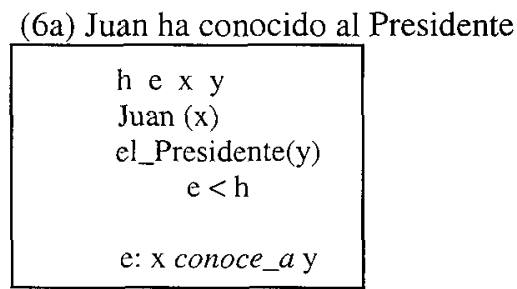

$\mathrm{K}_{6 \mathrm{a}}$

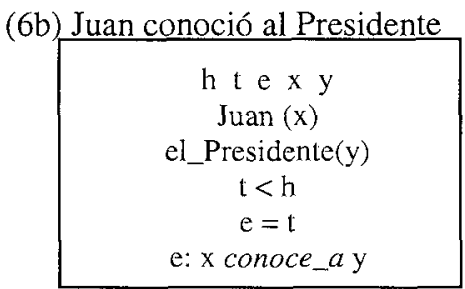

$K_{6 h}$

Dado que el instante $t$ de $\mathrm{K}_{6 \mathrm{~b}}$ no se asocia a ninguna condición, $t$ puede ser cualquier punto (es una variable libre) y, por tanto, las condiciones de verdad para ambas oraciones resultan idénticas. Creemos que esta aproximación no es adecuada ya que sí es posible distinguir ambos tiempos semánticamente, como veremos a continuación.

\subsubsection{Presente ampliado}

Alarcos (1970) y Dowty (1979) se apoyan fundamentalmente en la distribución de determinadas expresiones de tiempo con PC y PS para defender que lo característico del PC es que sitúa las eventualidades en un intervalo o marco temporal que incluye al presente:

\section{Expresiones PS}

ayer

anoche

la semana pasada en cierta ocasión en aquellos días el lunes pasado

\author{
Expresiones PC \\ hoy \\ hasta ahora \\ últimamente \\ estos días \\ recientemente \\ en los últimos 20 años \\ todavía no \\ ahora
}

\section{Expresiones PC y PS \\ esta mañana}

a las 10

A partir de estos datos Alarcos (1970) afirma que

«se emplea el perfecto compuesto (he cantado) con los adverbios que indican que la acción se ha efectuado en un periodo de tiempo en el que se haya comprendido el

\footnotetext{
${ }^{7}$ La representación se correspondería con la propuesta de Rojo. Reichenbach incluye un punto de referencia también para el PS. También ese punto de referencia sería una variable libre sin ninguna condición sobre él y por tanto no modificaría las condiciones de verdad.
} 
momento presente de que habla o escribe [...] Se emplea el perfecto simple (canté) con los adverbios que indican que la acción se produce en un periodo de tiempo en el que no está incluido el momento presente del que habla».

Esta propuesta puede formalizarse como sigue en términos de TRD:

(7a) Juan ha Ilegado hoy

\begin{tabular}{|l|}
\hline $\mathrm{h} \mathrm{T} e \mathrm{x}$ \\
$\mathrm{h} \subset \mathrm{T}$ \\
$\mathrm{e}<\mathrm{h}$ \\
$\mathrm{e} \subset \mathrm{T}$ \\
hoy $(\mathrm{T})$ \\
Juan(x) \\
$\mathrm{e}: \mathrm{x}$ llega \\
\multicolumn{1}{|}{$\mathrm{K}_{7 \mathrm{a}}$}
\end{tabular}

(7b) Juan llegó ayer

\begin{tabular}{|c|}
\hline $\mathrm{h} \mathrm{T} \mathrm{e} \mathrm{x}$ \\
$\mathrm{T}<\mathrm{h}$ \\
$\mathrm{e}<\mathrm{h}$ \\
$\mathrm{e} \subset \mathrm{T}$ \\
ayer $(\mathrm{T})$ \\
Juan(x) \\
$\mathrm{e:} \mathrm{x} \mathrm{Ilega}$ \\
\multicolumn{1}{|c}{$\mathrm{K}_{7 \mathrm{~b}}$}
\end{tabular}

$\mathrm{K}_{7 \mathrm{a}}$ puede parafrasearse así:

- Hay un evento $e$ (Juan llega)

- Este evento es anterior del momento del habla $h$

- Este evento ocurre dentro de un intervalo $T$ que también incluye al momento del habla.

En adelante llamaremos a este intervalo, T, marco temporal (MT). Un problema para esta propuesta es determinar si el MT existe en todos los casos. Esto es, si también es necesario incluirlo si no se menciona explícitamente. Igualmente debemos mostrar si tal marco temporal puede emplearse también con el PS (lo veremos en el apartado 3).

El siguiente ejemplo muestra que los hablantes emplean MMTT aunque no los mencionen de forma explícita. Supongamos que esta tarde María y sus padres han ido a una fiesta. En la fiesta María conoce a Juan. Ahora supongamos que la madre de María hace al padre de María esta pregunta: ¿Desde cuando conoce nuestra hija a ese chico?. El padre podría responder de una de estas formas: a) lo ha conocido en la fiesta, b) lo conoció en la fiesta. La respuesta escogida depende sólo de la distancia desde la eventualidad hasta $h$.

a) Si hace la pregunta estando aún en la fiesta, sólo podría usar el PC

b) Si ha acabado la fiesta pero están en el mismo día, podría usar PC o PS

c) Si la fiesta fue el día anterior, normalmente usaría el PS. 
Entendemos que este fenómeno sólo se puede explicar si asumimos que el oyente construye un marco temporal que, en el ejemplo, coincide con un día ${ }^{8}$.

\section{3. Conclusiones}

Podemos concluir este apartando afirmando que la única representación que nos permite obtener las condiciones de verdad de PC y PS es la que se basa en la propuesta del presente ampliado para el PC. Para formalizar semánticamente en términos de TRD la aportación de estos dos tiempos, proponemos que ambos establecen relaciones entre:

- h: momento del habla

-e: eventualidad descrita

-T: marco temporal

Las relaciones son:

PC: $\quad(\mathrm{h} \subset \mathrm{T}) \wedge(\mathrm{e}<\mathrm{h}) \wedge(\mathrm{e} \subset \mathrm{T})$

PS: $\quad(\mathrm{T}<\mathrm{h}) \wedge(\mathrm{e} \subset \mathrm{T})$

$\mathrm{Y}$ estas son las representaciones para el caso básico:

\begin{tabular}{|c|} 
Juan ha llegado \\
\hline $\mathrm{h} \mathrm{T} \mathrm{e} x$ \\
Juan $(\mathrm{x})$ \\
$\mathrm{h} \subseteq \mathrm{T}$ \\
$\mathrm{e}<\mathrm{h}$ \\
$\mathrm{e} \subseteq \mathrm{T}$ \\
$\mathrm{e} \cdot \mathrm{x}$ llega \\
\hline
\end{tabular}

\begin{tabular}{|c|}
\hline Juan llegó \\
\hline $\mathrm{h} T \mathrm{~T}$ \\
Juan $(\mathrm{x})$ \\
$\mathrm{T}<\mathrm{h}$ \\
$\mathrm{e} \subseteq \mathrm{T}$ \\
$\mathrm{e}: \mathrm{x}$ 1lega \\
\hline
\end{tabular}

\section{Extendiendo el análisis}

En este apartado veremos cómo podemos extender el análisis de PC y PS anterior para representar la interpretación de oraciones que incluyen expresiones que tienen efectos sobre la interpretación temporal.

\subsection{Negación}

Veíamos antes la dificultad que planteaba la teoría del estado resultante para explicar el efecto de la negación sobre el PC. Una de las ventajas de la representación que proponemos es que permite explicar con gran naturalidad el efecto de la negación tanto sobre PC como sobre PS:

\footnotetext{
${ }^{8}$ Comrie (1976, pág. 61) nota que en la gramática del S. XVII francés había una «regla de las 24 horas» que indicaba que el passé composé sólo debiera usarse cuando lo descrito hubiera ocurrido en las últimas 24 horas. Igual que en el francés del S. XVII, en el español peninsular actual hay una fuerte tendencia a asociar el PC con ese intervalo de tiempo precisamente.
} 
(8a) Juan no ha estado en Madrid

(8b) Juan no estuvo en Madrid

Tanto en (8a) como en (8b) se afirma la existencia de un lapso de tiempo (MT) durante el cual un suceso no llega a producirse. Esto es lo que captan las siguientes representaciones:

(8a) Juan no ha estado en Madrid

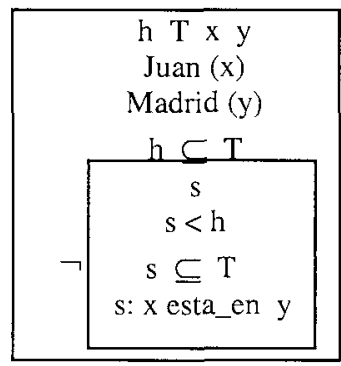

(8b) Juan no estuvo en Madrid

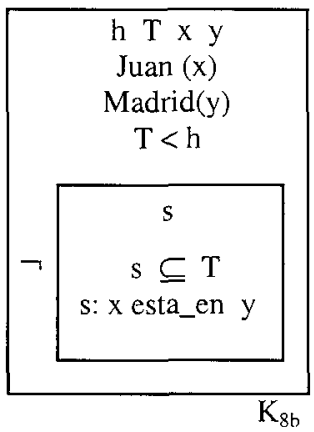

Además, esta representación es fácilmente adaptable a la hipótesis de que la negación de un evento o estado es un estado ${ }^{9}$. Ciertamente no tenemos más que suponer que el estado es el descrito por la caja negada:
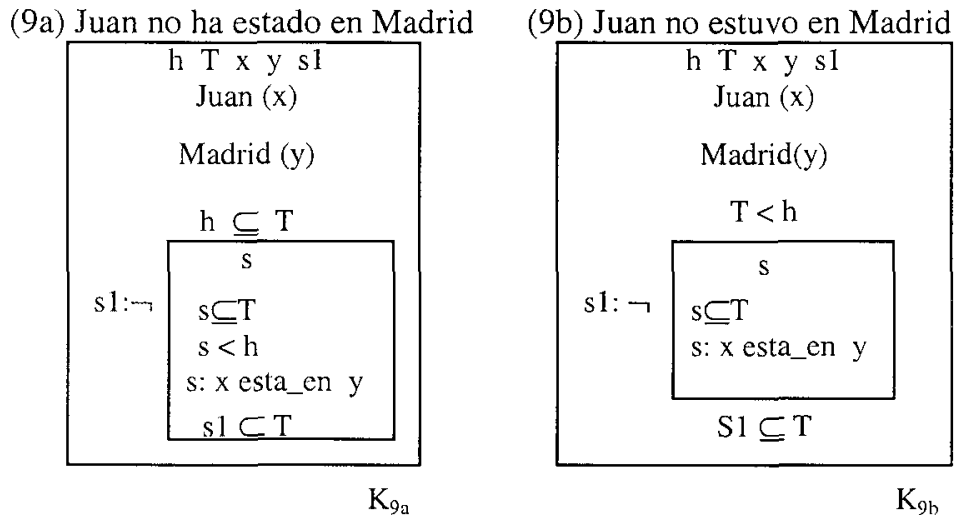

\footnotetext{
${ }^{9}$ Propuesta por A. Molendijk y H. de Swart (1997).
} 


\section{2. «Nunca»}

«Nunca» puede interpretarse como un adverbio de frecuencia. Compárese:

Juan no ha venido nunca ( 0 veces)

Juan ha venido dos veces ( 2 veces)

Juan ha venido muchas veces (muchas veces)

No obstante, si nos limitamos a considerarlo un adverbio temporal no podemos explicar las diferencias entre:

Juan no ha venido ( 0 veces)

Juan no ha venido nunca ( 0 veces)

Estas diferencias podemos explicarlas si suponemos que «nunca» tiene una doble función: especifica el tamaño de T (adverbio temporal) y cuantifica:

Juan no ha venido hoy ( $\mathrm{T}=1 \mathrm{dí}$ )

Juan no ha venido esta semana ( $\mathrm{T}=1$ semana)

Juan no ha venido este año ( $\mathrm{T}=1$ año)

Juan no ha venido nunca ( $\mathrm{T}=$ desde que nació)

Así, «nunca» maximiza el tamaño de $T$.

Esto explicaría que muchos hablantes no acepten «nunca» con otro adverbio temporal:

* Aquel verano, Paco nunca construyó casas

* En los próximos años, Paco nunca construirá casas

Veamos para terminar este punto las representaciones para:

(10a) Juan no ha venido hoy

(10b) Juan no ha venido nunca

(10a) Juan no ha venido hoy

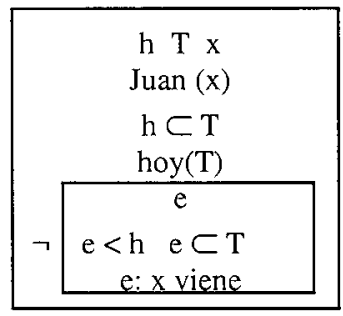

(10b) Juan no ha venido nunca

$$
\mathrm{K}_{10 \mathrm{a}}
$$

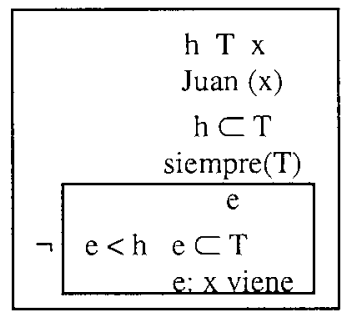

$\mathrm{K}_{10: 2}$ 


\subsection{Hoy/ayer}

Estos adverbios añaden una condición sobre el marco temporal T. Si el conjunto de condiciones sobre $\mathrm{T}$ son incompatibles, entonces la oración será semánticamente incorrecta. Veamos algunos ejemplos:

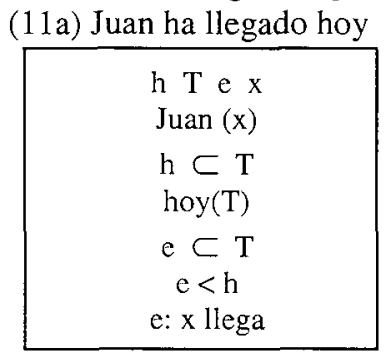

$\mathrm{K}_{11 \mathrm{a}}$

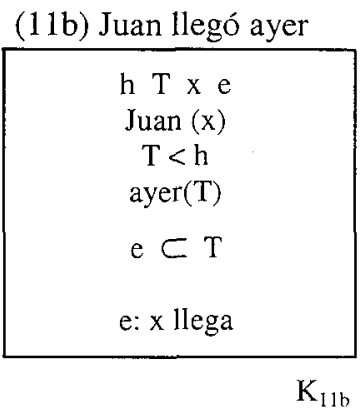

La representación para *Juan llegó hoy sería:

$$
\begin{gathered}
\text { h } T x \text { e } \\
\text { Juan }(x) \\
T<h \\
\text { hoy }(T) \\
e \subset T \\
\text { e: } x \text { llega }
\end{gathered}
$$

Dado que las condiciones sobre $\mathrm{T}(\operatorname{hoy}(T)$ y $T<h)$ son incompatibles, la oración es semánticamente incorrecta.

\subsection{Antes...}

Una expresión como «antes...» introduce un referente temporal $(t)$ que es anterior temporalmente a la eventualidad, pero que queda situado dentro del marco temporal. Sean los siguientes ejemplos:

(12a) Juan ha llegado antes de las 9

(12b) Juan llegó antes de las 9

La relación entre $h$ y las 9 varía de un caso a otro. Con PS (12b), parece claro que las 9 es anterior a $h$. Por el contrario, con PC (12a) las 9 puede ser anterior o posterior a $h$. Este fenómeno se debe a que forzamos que el referente temporal esté situado dentro de $T$ y, dado que $h$ está incluido en $T$, es posible que $t$ quede detrás de $h$. 
(12a) Juan llegó antes de las 9

\begin{tabular}{|c|}
\hline $\mathrm{h} \mathrm{T} e \mathrm{xt}$ \\
$\operatorname{Juan}(\mathrm{x})$ \\
$\mathrm{T}<\mathrm{h}$ \\
las $9(\mathrm{t}) \mathrm{t} \subset \mathrm{T}$ \\
$\mathrm{e}<\mathrm{t}$ \\
$\mathrm{e} \subset \mathrm{T}$ \\
$\mathrm{e}: \mathrm{x} l 1 e g a$ \\
\hline
\end{tabular}

(12b) Juan ha llegado antes de las 9

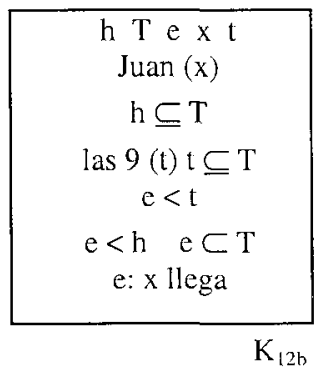

Nuevamente vemos que las cajas obtenidas captan la representación adecuada.

\section{Análisis del discurso}

Una vez obtenidas las posibles representaciones semánticas de las oraciones independientes podemos proceder a estudiar cuáles son las relaciones que en el discurso mantienen tales oraciones. Nuestro objetivo es ver de qué forma restringen o intervienen PC y PS a la hora de calcular las relaciones discursivas.

Para ello debemos empezar por ver cuáles son las posibles relaciones temporales. Kamp y Reyle (1993) distinguen tres posibles relaciones temporales entre dos eventualidades $(\mathrm{e} 1, \mathrm{e} 2)$ descritas en dos oraciones consecutivas:

a) Escenario: e2 es un estado que se da anteriormente a el $(\mathrm{e} 1 \subset \mathrm{e} 2)$

Juan entró en la habitación. (e1) La habitación estaba oscura. (e2)

b) Narración: e2 sigue a el $($ el $<$ e2)

Juan cogió un vaso de cerveza. (e1) Se lo bebió de un trago.(e2)

c) Explicación: e2 es anterior a el (e2<e1)

Juan entró en el bar. (e1) Había oído un ruido.(e2)

A estas relaciones podemos añadir una cuarta (mencionada entre otros por Asher y Lascarides, 1993):

d) Elaboración: e2 describe una parte de e1 $(\mathrm{e} 2 \subset \mathrm{e} 1)$

Los niños construyeron un castillo de arena. (e1) Luis hizo la torre. (e2) 
Junto a estas relaciones temporales entre eventos, podemos plantearnos la pregunta de si se dan relaciones en las que intervienen los marcos temporales. Lo veremos en los siguientes epígrafes.

\subsection{Relaciones temporales entre eventos}

La siguiente tabla recoge las posibles relaciones temporales entre dos eventos (e1, e2) descritos ambos con PC o ambos con PS.

\begin{tabular}{|c|c|c|}
\hline Rel. discursiva & $\mathrm{PC}+\mathrm{PC}$ & PS+PS \\
\hline $\begin{array}{l}\text { Narración } \\
(\mathrm{e} I<\mathrm{e} 2)\end{array}$ & $\begin{array}{l}\text { Hoy me he levantado a las } 7 \text { y he } \\
\text { desayunado en la cama. }\end{array}$ & $\begin{array}{l}\text { Esta mañana me levanté a las } \\
7 \text { de la mañana y desayuné } \\
\text { en la cama. }\end{array}$ \\
\hline $\begin{array}{l}\text { Elaboración } \\
\text { (e1 } \supset \text { e2) }\end{array}$ & $\begin{array}{l}\text { Juan se ha comprado una casa. } \\
\text { Su hermano le ha arreglado el } \\
\text { papeleo. }\end{array}$ & $\begin{array}{l}\text { Juan se compró una casa. Su } \\
\text { hermano le arregló todo el } \\
\text { papeleo. }\end{array}$ \\
\hline $\begin{array}{l}\text { Escenario } \\
(\mathrm{e} 1 \subset \mathrm{e} 2)\end{array}$ & & - \\
\hline $\begin{array}{l}\text { Retroceso } \\
(\mathrm{e} 2<\mathrm{e} 1)\end{array}$ & $\begin{array}{l}\text { Juan se ha comprado una casa. } \\
\text { Le ha tocado la lotería. }\end{array}$ & $\begin{array}{l}\text { Juan se compró una casa en } \\
\text { París. Le tocó la lotería. }\end{array}$ \\
\hline
\end{tabular}

La tabla muestra que los pares $\mathrm{PC}+\mathrm{PC}$ y $\mathrm{PS}+\mathrm{PS}$ se comportan de forma simétrica. Ambos pares pueden aparecer en secuencias narrativas, elaborativas $O$ explicativas (retroceso), pero ninguno de ellos puede actuar de escenario.

La siguiente tabla recoge las posibles relaciones temporales entre dos eventos (e1, e2) descritos por dos enunciados consecutivos, uno de los cuales aparece en PC y otro en PS.

\begin{tabular}{|l|l|l|}
\hline Rel. discursiva & $\mathbf{P C}+\mathbf{P S}$ & PS+PC \\
\hline $\begin{array}{l}\text { Narración } \\
(\mathrm{e} 1<\mathrm{e} 2)\end{array}$ & - & $\begin{array}{l}\text { Esta mañana me levanté a las } \\
\text { 7. Durante todo el día he } \\
\text { trabajado en casa. }\end{array}$ \\
\hline $\begin{array}{l}\text { Elaboración } \\
(\mathrm{e} 1 \supset \mathrm{e} 2)\end{array}$ & $\begin{array}{l}\text { Hoy hemos trabajado mucho. } \\
\text { Estuvimos primero en la obra y } \\
\text { luego... }\end{array}$ & - \\
\hline $\begin{array}{l}\text { Escenario } \\
(\mathrm{e} 1 \subset \mathrm{e} 2)\end{array}$ & - & - \\
\hline $\begin{array}{l}\text { Retroceso } \\
(\mathrm{e} 2<\mathrm{e} 1)\end{array}$ & $\begin{array}{l}\text { Juan se ha comprado una casa. } \\
\text { Le tocó la lotería. }\end{array}$ & - \\
\hline
\end{tabular}

Podemos ver que en este caso su comportamiento es bien diferente. Mientras que el PS puede explicar o elaborar al PC, el PC sólo puede mantener una relación 
de narración con un PS anterior. Dado que se oponen como pasado/presente, es natural que la relación PC+PS pueda ser de retroceso (y no de narración) mientras que la relación $\mathrm{PS}+\mathrm{PC}$ pueda ser de narración (y no de retroceso).

Con respecto a la relación de elaboración para PC+PS, es una estructura estilística típica de la prensa escrita. El PC se adapta perfectamente a la presentación de la noticia (relevancia actual), pero la mayor precisión asociada al PS la hace más adecuada a la narración de los sucesos concretos ${ }^{10}$.

Veamos la representación obtenida para dos enunciados cuyos eventos mantienen una relación de elaboración:

(13) Juan fue ayer a Madrid. Se compró un disco.

\begin{tabular}{|c|}
\hline h T e $x$ y \\
z u e \\
T $<$ ayer $(T)$ \\
Juan $(x)$ Madrid (y) \\
e $\subset \mathrm{T}$ \\
e: $x$ ir_a y \\
z=x disco(u) \\
e' $\subset \mathrm{T}$ \\
e' $\subset$ e \\
e': $z$ compra u \\
\hline
\end{tabular}

\subsection{Relaciones temporales en las que intervienen los MMTT}

Cuando hemos establecido la representación de PC y PS hemos incluido junto a la eventualidad un marco temporal. En consecuencia podemos preguntarnos si a las relaciones entre eventualidades es preciso añadir relaciones de un nivel superior entre marcos temporales. Creemos que eso es precisamente lo que encontramos en textos como los siguientes:

(14) El IPC subió en marzo un 0,3\%. En lo que va de año, el IPC ha subido un $1,4 \%$.

(15) Los gamberros destrozaron cabinas y quemaron coches y contenedores (14) es un ejemplo de la relación que llamaremos enfoque. (15) es un ejemplo de la relación que llamaremos resumen.

\footnotetext{
${ }^{10}$ El siguiente ejemplo proviene del diario El País ( 6 de enero de 1998):

$X$, de 21 años, ha confesado que mató a... X declaró haber actuado inducida por su compañero sentimental, José E. A., de 27 años, que no es el padre de los niños.

No hay justificación aparente en el salto del PC (ha confesado) al PS (declaró) más que la preferencia por el PC al inicio de la noticia y el PS en la exposición.
} 


\subsubsection{Enfoque}

En (14) no hay una relación de narración ni de retroceso (los eventos no se siguen); tampoco podemos decir que el segundo evento (subir un 1,4\%) sea el escenario en el que ocurre el otro. Más bien se trata de un cambio de punto de vista $\mathrm{o}$, en nuestros términos, un cambio de marco temporal. El hablante sitúa su segundo enunciado en un MT mucho más amplio desde el que puede dar una imagen global.

Se trata de una estructura discursiva relativamente frecuente en los periódicos. Con ella a menudo el autor puede extraer conclusiones o presentar hechos globales o relevantes (que quiere destacar especialmente). Veamos otro ejemplo:

Terroristas del comando Vizcaya de ETA intentaron asesinar a Pérez Aja mientras paseaba con su perro cerca de su domicilio. Dos jóvenes se le acercaron por la espalda y uno de ellos le descerrajó un tiro que le entró por detrás de la oreja y le salió por el maxilar. Los terroristas hicieron explotar el coche en el que emprendieron la huida.

En lo que va de año, ETA ha perpetrado 12 atentados con víctimas, en los que han resultado muertas un total de 9 personas. Cinco de estos atentados, con tres muertos, son responsabilidad del comando Vizcaya. (Fuente: Diario El País, 11 de Junio de 1997)

El texto se inicia con una presentación del suceso seguida de una narración en PS. En el segundo párrafo, el autor pasa del hecho puntual al escenario global, amplía su perspectiva para mostrarnos lo general en lugar de lo específico. Los eventos descritos no pueden relacionarse directamente ya que cada uno parece pertenecer a un marco temporal diferente, se relacionan a través de su marco temporal.

Veamos a continuación la representación en TRD obtenida para el primer ejemplo que recogíamos: 
(14) El IPC subió en marzo un $0,3 \%$. En lo que va de año, el IPC ha subido un $1,4 \%$.

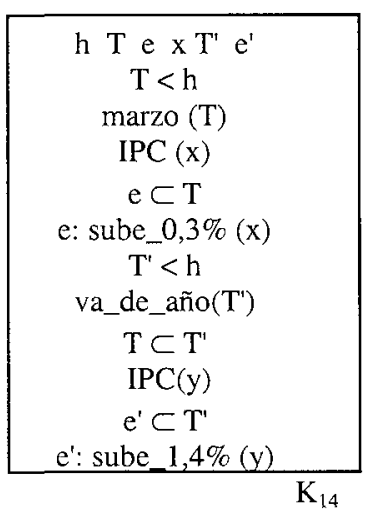

\subsubsection{Resumen}

Retomemos ahora el ejemplo (15):

(15) Los gamberros destrozaron cabinas y quemaron coches y contenedores

Para entender la oración (17), el orden no es relevante y posiblemente el propio hablante no lo conoce. Más bien lo que interesa en este caso es indicar que una serie de sucesos han ocurrido en un determinado marco temporal.

Podemos representar estos enunciados como una serie de eventos relacionados por el hecho de estar en un mismo marco temporal, pero sin especificar las relaciones entre ellos ${ }^{11}$. En términos del metalenguaje de la TRD obtendríamos lo siguiente:

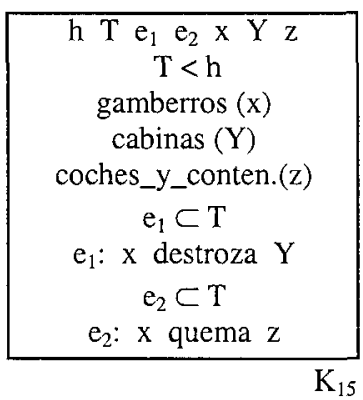

${ }^{11}$ Cfr. a este respecto al artículo de L. de Saussure (1996). 


\section{Conclusiones}

En este artículo hemos examinado desde la perspectiva de una semántica veritativa el significado dos tiempos verbales del español: PC y PS. Hemos revisado algunos análisis previos sobre la cuestión $\mathrm{y}$, hemos comparado las diferentes propuestas expresándolas en términos de condiciones de verdad. Nuestro análisis nos ha llevado a apoyar la teoría del presente ampliado.

La representación obtenida a partir de esta teoría capta con gran naturalidad tanto los casos básicos como aquellas oraciones en las que aparecen un adverbio de negación o diversas expresiones temporales.

Además, partiendo de tal representación podemos describir diversas relaciones discursivas. Estas relaciones no se dan sólo entre oraciones, sino también entre los marcos temporales (introducidos de acuerdo con la teoría del presente ampliado).

\section{Referencias bibliográficas:}

Alarcos Llorach, E. (1970): Estudios de Gramática Funcional del español. Madrid: Gredos.

Asher, N. y A. Lascarides, (1993): «Temporal interpretation, discourse relations and commonsense entailment», Linguistics and Philosophy, 16: págs. 437-493.

Bello, A. (1860): Gramática de la lengua castellana. Madrid: Edaf, 1978.

Carey, K. (1996): «From Resultatives to Current Relevance: English and Modern Castillian Spanish», in A. E. Goldberg (ed.) Conceptual Structure, discourse and language, CSLI.

Comrie, B. (1976): Aspect, Cambridge: CUP.

Dowty, D. (1979): Word Meaning and Montague Grammar. Dordrecht: Reidel.

García Fernández, L. (1995): «La interpretación temporal de los tiempos compuestos», Verba, 22, págs. 363-396.

Harder, P. (1996): Functional Semantics: A Theory of Meaning, Structure and Tense in English, Berlin: Mouton de Gruiter.

Hovy, E. y E. Meier (1992): «Organizing discourse structure relations using metafunctions», in H. Horazek y M. Zock (eds.) New Concepts in Natural Language Generation, págs. 57-68.

Kamp, H y U. Reyle. (1993): From Discourse to Logic. Dordrecht: Kluwer.

Luscher, J. M. y B. Sthioul, (1996): «Emplois et interpretations du Passé Compossé», Cahiers de Linguistique française, 18, págs. 187-217.

Molendijk, A., (1994): «Tense use and temporal orientation: The passé simple and the imparfait of french», in Co Vet \& Car Vetters (eds.): Trends in Linguistics, 75 Tense and Aspect in Discourse, Berlin: Mouton de Gruiter, págs. 5-20. 
Montague, R. (1974): Formal Philosophy: selected papers of Richard Montague. (ed.) R. H. Thomason, New Haven: Yale University Press.

Moreno Cabrera, J. C. (1994): Curso Universitario de Lingüística general. Tomo 1: Teoría de la gramática y sintaxis general, Madrid: Síntesis.

Reichenbach, H. (1947): Elements of symbolic logic. Londres y Nueva York: MacMillan.

Rodríguez, Y. (1997): Adjuntos de localización temporal: estructura e interpretación, Tesis doctoral, Universidad Autónoma de Barcelona

Rojo, G. (1974): «La temporalidad verbal en español», Verba, 1, págs. 69-149.

Rojo, G. (1990): «Relaciones entre temporalidad y aspecto en el verbo español», en I. Bosque (ed.): Tiempo y Aspecto en Español, Madrid: Cátedra, págs. 17-43.

Saussure, L. de, (1996): «Encapsulation et référence temporelle d'énoncés négatifs au passé composé et au passé simple», Cahiers de Linguistique Française, 18, págs. 219-42

Vendler, Z. (1957): «Verbs and Times», The Philosophical Review, 66, págs, 195220.

Vet, C. (1980): Temps, aspect et adverbs de temps en français comtemporaine.

Vidal Villalba, J. (1992): Tratamiento del tiempo y del aspecto, Tesis doctoral inédita, Universidad de Barcelona. 